\title{
Preparation and Performance of cis-Polybutadiene Rubber Composite Materials Reinforced by Organic Modified Palygorskite Nanomaterials
}

\author{
Fei Wang, ${ }^{1,2}$ Lei Feng, ${ }^{3}$ Qingguo Tang, ${ }^{1}$ Haifeng Liu, ${ }^{1}$ and Huimin Liu ${ }^{1}$ \\ ${ }^{1}$ Institute of Power Source \& Ecomaterials Science, Hebei University of Technology, Tianjin 300130, China \\ ${ }^{2}$ Key Laboratory for Palygorskite Science and Applied Technology of Jiangsu Province, Huaiyin Institute of Technology, \\ Huaian 223003, China \\ ${ }^{3}$ Qianian College, Hebei United University, Tangshan 064400, China \\ Correspondence should be addressed to Fei Wang; wangf777@yeah.net
}

Received 24 September 2013; Accepted 2 November 2013

Academic Editor: Jianping Xie

Copyright $\odot 2013$ Fei Wang et al. This is an open access article distributed under the Creative Commons Attribution License, which permits unrestricted use, distribution, and reproduction in any medium, provided the original work is properly cited.

\begin{abstract}
The hydrophilic character of palygorskite has been modified by grafting organic group and controlling surface energy for improving compatibility of palygorskite in rubber matrix using palygorskite as cis-polybutadiene rubber fillers. The effects of coupling modification on the performance of cis-polybutadiene rubber materials filled with palygorskite were investigated, and the influence of coupling agent dosage on their mechanical properties was also studied. The results indicated that the mechanical performance of cis-polybutadiene rubber materials reinforced by modified palygorskite could be improved significantly, and the tensile strength and tearing strength increased by $122.5 \%$ and $107.6 \%$ at the optimal dosage (15\%) of coupling agent 3-mercaptopropyl trimethoxysilane. Moreover, the reinforcement mechanism of rubber composite materials as prepared was also analyzed.
\end{abstract}

\section{Introduction}

cis-Polybutadiene rubber materials have the characteristics of wear resistance, excellent elastic, age resistance and so forth, and thus they have become essential in many fields [1-3]. Nevertheless, cis-polybutadiene rubber materials as organic materials could not form crystals at room temperature unless they are sufficiently stretched and have disadvantages of low compressive strength, high cost, and poor dimensional stability, and thus their stress-induced crystallization is obviously lower than that of natural rubber $[4,5]$. Consequently, fillers have been widely used in rubber products, which could enhance the performance of rubber composites and reduce the production cost. The carbon black is the prime fillers in rubber industry, whose process exacerbates tensions in the energy supply and pollutes the environment. The studies on energy saving and environment-friendly fillers have focused on polymer composites along with the emphasis on the environmental protection.
Natural mineral materials such as sepiolite, montmorillonite, and kaolin have been applied as fillers of polymers for various composite materials with excellent performance [611], because applying natural mineral materials will solve the serious environmental problem caused by carbon black production and so forth. Palygorskite is typical natural fibrillar silicate clay with large reserves in South China. The chemical structure of palygorskite is $\mathrm{Mg}_{5} \mathrm{Si}_{8} \mathrm{O}_{20}(\mathrm{OH})_{2}\left(\mathrm{H}_{2} \mathrm{O}\right)_{4} \cdot 4 \mathrm{H}_{2} \mathrm{O}$ and its smallest structure unit is fibrillar single crystal with a diameter of $20-40 \mathrm{~nm}$ [12-15]. Unlike the layerlayer interaction in layered silicates, the interaction between palygorskite single crystals is extremely weak due to similar line-line contact, and voluminous interstitial spaces are in these agglomerated single crystals [16, 17]. Palygorskite as a kind of filler has hydrophilic characteristics, which limit the dispersibility of mineral powder in rubber matrix and the application of mineral fillers in rubber industry. Surface modification is widely applied to overcoming this limitation of mineral fillers. The first method is performed 
based on polar functional oligomer. The second method is carried out through predispersing clay compatible with cispolybutadiene rubber. The main purpose of the above two methods is to improve interfacial interaction, and thus the properties of nanocomposites are strongly influenced by the nature of filler-matrix interface [18-21]. However, there is only a few reports about cis-polybutadiene rubber nanocomposites containing modified palygorskite nanomaterials.

The objective of this work is to develop the cispolybutadiene rubber composite materials using palygorskite minerals as reinforced fillers. The strong interaction caused by the coupling reaction could improve the dispersity effect of the palygorskite in the cis-polybutadiene rubber matrix, and the corresponding properties of the resulting nanocomposites are also systematically investigated.

\section{Experimental}

The palygorskite as one of natural fibrillar minerals was supplied by Jiuchuan Nanomaterials Technology Co., Ltd. The titanate coupling agent, NDZ-201(isopropyl tri(dioctylpyrophosphate) titanate) and silane coupling agents, SGSi998 (bis [-(triethoxysilyl) propyl] disulfide), KH560 ( $\gamma$ (2,3-epoxypropoxy) propyltrimethoxysilane), KH570 (3(methacryloyloxy) propyltrimethoxysilane), and KH590 (3-mercaptopropyltrimethoxysilane) were supplied by Shuguang Chemical Group Co., Ltd. The cis-polybutadiene rubber was supplied by Yanshan Petrochemical Co., Ltd. The compounding ingredients were purchased from chemical stores.

Under the condition of acid, silane coupling agent hydrolysis was performed by means of mixing silane coupling agent in the water solution of ethanol. Then, an appropriate amount of palygorskite was added to the mixture with vigorous stirring at $70^{\circ} \mathrm{C}$. The products were filtered and washed several times with deionized water. The filter cake was taken out and then dried by airing. In order to remove water sufficiently, the modified palygorskite was ground using a mortar and a pestle and then dried at $110^{\circ} \mathrm{C}$ in oven until the weight was not changed. The rubber composites filled with palygorskite minerals were prepared. cis-Polybutadiene rubber, modified palygorskite minerals, and other compounding ingredients such as zinc oxide, stearic acid, zinc oxide, accelerator CZ, antioxidant $\mathrm{RD}$, emollient, and sulphur were mixed using a laboratory-sized two-roll mill. The optimum cure time ( $t 90)$ which is the time for the completion of cure was determined at $145^{\circ} \mathrm{C}$ using a curometer. The above composites were vulcanized at platen press with $13 \mathrm{MPa}$ pressure, based on the t90 values.

The contact angle of powder was measured using capillary penetration measurements. In this work, the powder was placed in a glass tube with a filter on the bottom. The tube was attached to an electrobalance (DataPhysics DCAT21), which could record the weight gain as a function of time when the bottom of the tube touched the testing solvent. On the above basis, the surface free energy values were calculated using the contact angles of three kinds of liquid by Wu's equation $[22,23]$. The microstructure of the samples was observed by

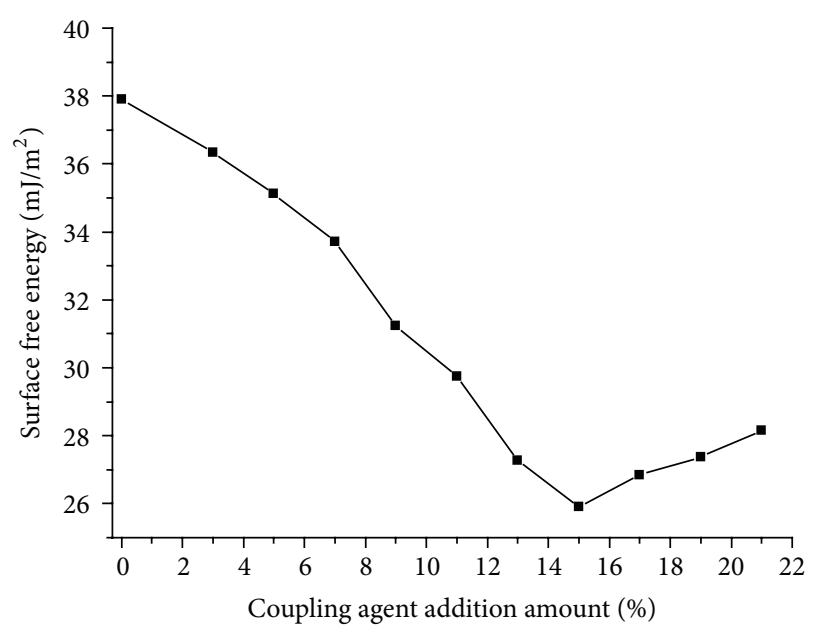

FIGURE 1: Variation of palygorskite surface free energy after modification with different coupling agent (3-mercaptopropyl trimethoxysilane) addition amounts.

scanning electron microscopy (Philips XL30) at $25.0 \mathrm{kV}$ and $30 \mu \mathrm{A}$. Dumb-bell shaped specimens were punched from the moulded sheets by a tensile specimen cutter. Tensile strength and elongation at break were measured following GB/T 5281998 using a universal tensile testing machine (CMT6104).

\section{Results and Discussion}

Figure 1 shows the variation of surface free energy of palygorskite after modification with different coupling agent (3mercaptopropyl trimethoxysilane) addition amounts. From Figure 1, it can be seen that the palygorskite after modification with the coupling agent addition amount of $15 \%$ has the lowest surface free energy. When powders are dispersed into media, the lower surface free energy has positive influence on their dispersion [24-26]. According to the above analysis, $15 \%$ is selected as the coupling agent addition amount for the palygorskite in order to obtain the best modification and dispersion effect of palygorskite in the composite materials.

To study the influence of coupling agent for palygorskite on mechanical properties of cis-polybutadiene rubber composite materials, five kinds of coupling agent for palygorskite are chosen based on the above optimal coupling agent (3-mercaptopropyl trimethoxysilane) addition amount. The tearing strength results of rubber composite materials are shown in Figure 2. From Figure 2, we can see that the tearing strength values of rubber composite materials filled with different kinds of coupling agent modified palygorskite are close to each other. Among them, rubber composite materials filled with isopropyl tri(dioctylpyrophosphate) titanate modified palygorskite have the best tearing strength. The reason for the phenomenon is mainly that isopropyl tri(dioctylpyrophosphate) titanate belongs to lipid coupling agent, which has relatively long chain and good toughness $[27,28]$.

Figure 3 shows the tensile strength results of cispolybutadiene rubber composite materials. From Figure 3, 


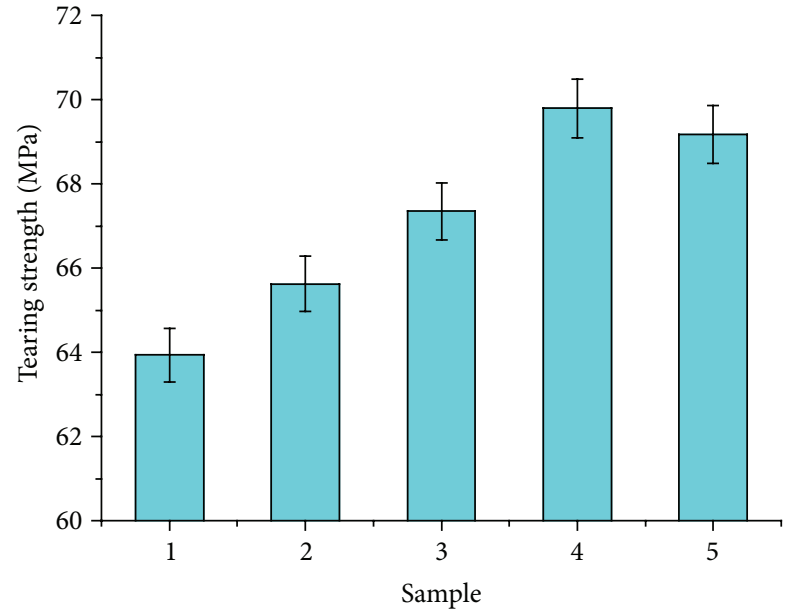

FIGURE 2: Effect of coupling agent for palygorskite on tearing strength of rubber composite materials (1: KH-570, 2: KH-560, 3: KH-590, 4: NDZ-201, 5: Si-998; errors in tearing strength values $(y$ axis) are designated as the vertical error bars).

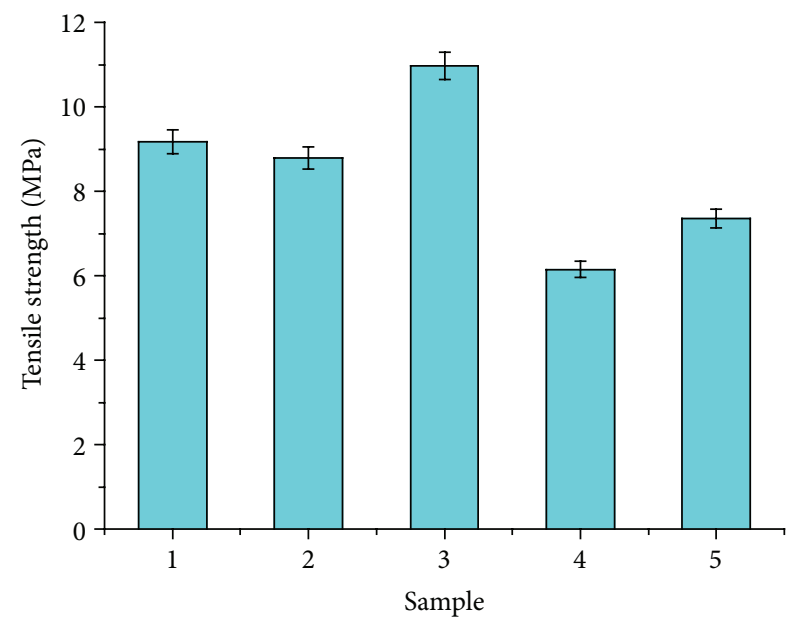

FIGURE 3: Effect of coupling agent for palygorskite on tensile strength of rubber composite materials (1: KH-570, 2: KH-560, 3: KH-590, 4: NDZ-201, 5: Si-998; errors in tensile strength values ( $y$ axis) are designated as the vertical error bars).

it can be seen that the tensile strength of rubber composite materials filled with different kinds of coupling agent modified palygorskite is different obviously. Among them, rubber composite materials filled with isopropyl tri(dioctylpyrophosphate) titanate modified palygorskite have the lowest tensile strength. The reason for the phenomenon is mainly that isopropyl tri(dioctylpyrophosphate) titanate belongs to lipid coupling agent, which has physical winding rather than chemical bond. The above results indicate that coupling agent modified palygorskite could enhance the mechanical properties of rubber composite materials effectively. Moreover, rubber composite materials filled with 3-mercaptopropyl trimethoxysilane modified palygorskite have the highest tensile strength. As shown in Figure 2, the rubber composite materials filled with isopropyl tri(dioctylpyrophosphate) titanate

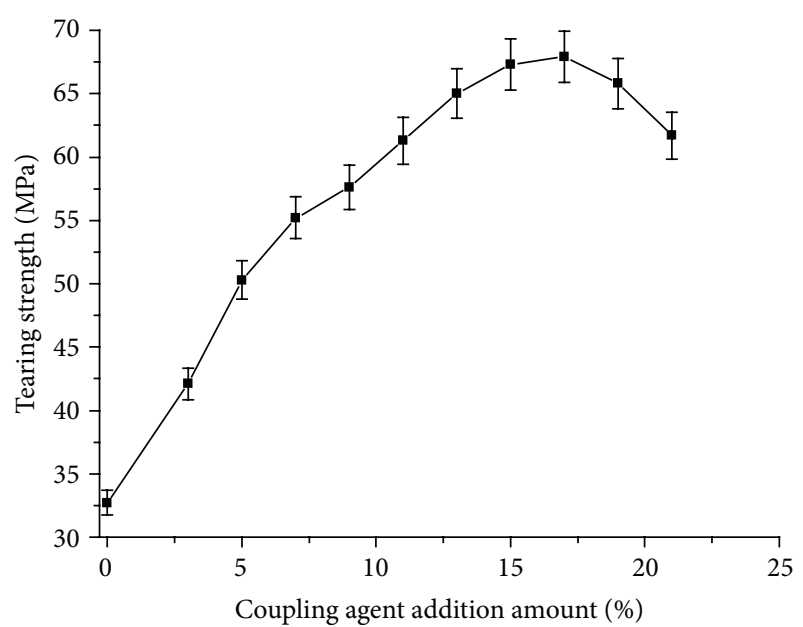

FIGURE 4: Variation of tearing strength of cis-polybutadiene rubber composite materials reinforced by palygorskite modified by different coupling agent (3-mercaptopropyl trimethoxysilane) addition amounts (errors in tearing strength values ( $y$-axis) are designated as the vertical error bars).

and isopropyl tri(dioctylpyrophosphate) titanate modified palygorskite have similar tearing strength. Therefore, 3-mercaptopropyl trimethoxysilane is chosen as the optimal coupling agent.

Figure 4 shows the variation of tearing strength of cispolybutadiene rubber composite materials reinforced by palygorskite modified by different coupling agent addition amounts. From Figure 4, we can see that the tearing strength values of rubber composite materials filled with different coupling agent addition amounts are different obviously. Among them, rubber composite materials filled with 17\% 3mercaptopropyl trimethoxysilane modified palygorskite have the best tearing strength.

Figure 5 shows the tensile strength results of cispolybutadiene rubber composite materials. From Figure 5, we can see that the tensile strength values of rubber composite materials filled with different coupling agent addition amounts of modified palygorskite are different obviously. Among them, rubber composite materials filled with 15\% 3mercaptopropyl trimethoxysilane palygorskite have the best tensile strength. As shown in Figure 4, the rubber composite materials filled with $15 \%$ and $17 \%$ 3-mercaptopropyl trimethoxysilane modified palygorskite have similar tearing strength. Therefore, 15\% 3-mercaptopropyl trimethoxysilane is chosen as the optimal coupling agent amount.

It is known that the dispersion of a filler in the polymer matrix can have a significant effect on the mechanical properties of the composites, and good dispersion can be achieved by surface modification of the filler particles and appropriate processing conditions [29-31]. The dispersibility of modified palygorskite in the cis-polybutadiene rubber matrix is confirmed by SEM shown in Figure 6, the dispersibility of modified palygorskite in the cis-polybutadiene rubber matrix is improved obviously compared with that of the unmodified 


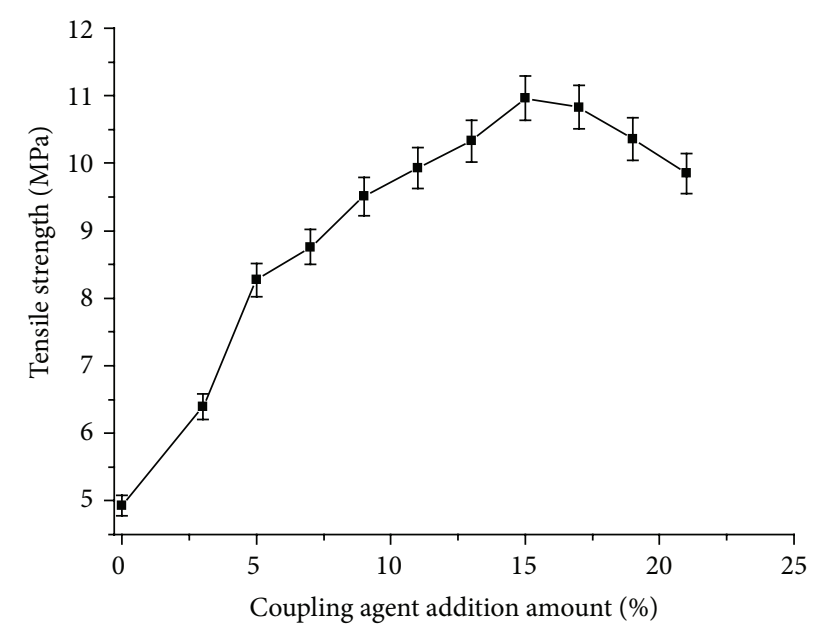

FIGURE 5: Variation of tensile strength of cis-polybutadiene rubber composite materials reinforced by palygorskite modified by different coupling agent (3-mercaptopropyl trimethoxysilane) addition amounts (errors in tensile strength values ( $y$-axis) are designated as the vertical error bars).

ones (Figure 6(a)), and the average diameter of modified palygorskite is much less than $100 \mathrm{~nm}$.

In the case of nanocomposites containing 15\% 3-mercaptopropyl trimethoxysilane modified palygorskite, most palygorskite nanofibers aggregates are broken down to primary particles, which could maximize the interfacial interaction between the palygorskite and the polymer matrix. Accordingly, the main reason for the obvious enhancement of mechanical performance lies in the good dispersion of modified palygorskite nanofibers in the cis-polybutadiene rubber matrix at a nanometer scale and the strong interaction between palygorskite and cis-polybutadiene rubber, which exhibits nanometer effect and physical cross-link of palygorskite. Due to the transferring stress and limiting the expansion of palygorskite cracks, the modified palygorskite nanomaterials could improve the mechanical properties of cis-polybutadiene rubber.

\section{Conclusions}

In this paper, the hydrophilic character of palygorskite was modified by grafting organic group and controlling surface energy for improving compatibility of palygorskite in rubber matrix, and the palygorskite minerals as prepared were used as cis-polybutadiene rubber fillers. The results showed that the mechanical properties of cis-polybutadiene rubber composite materials reinforced by modified palygorskite could be improved obviously. When the optimum dosage of coupling agent 3-mercaptopropyl trimethoxysilane was $15 \%$, the tensile strength and tearing strength increased by $122.5 \%$ and $107.6 \%$, respectively. The reason for the above phenomenon was that nanometer effect and physical crosslink of palygorskite nanofibers shown in the microstructure of cis-polybutadiene rubber composite materials fracture surface could maximize the interfacial interaction between polymer matrix and palygorskite.

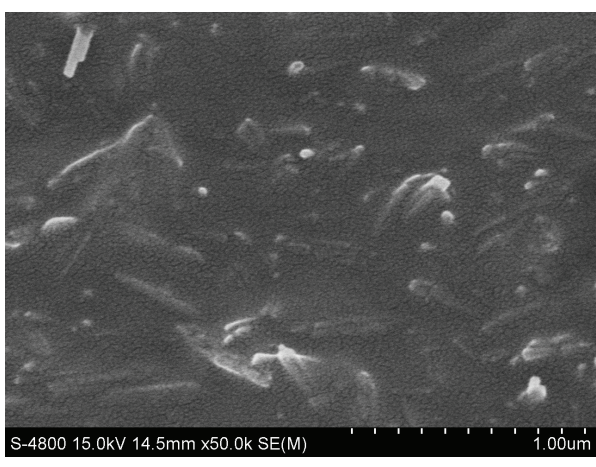

(a)

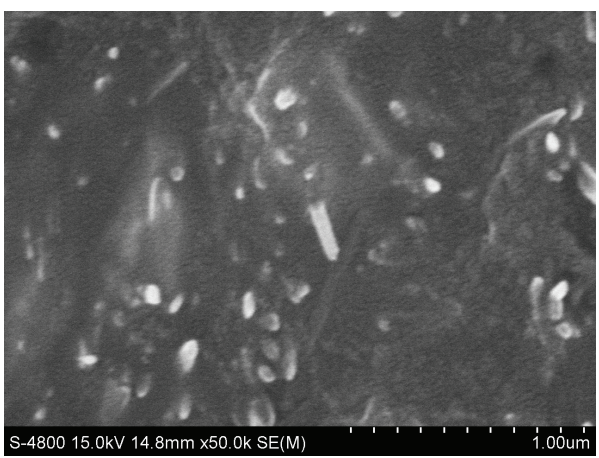

(b)

FIGURE 6: SEM micrograph of fracture surface of cis-polybutadiene rubber composites ((a) composites containing unmodified palygorskite samples and (b) composites containing 15\% 3-mercaptopropyl trimethoxysilane modified palygorskite).

\section{Acknowledgments}

This research was financially supported by the Application Foundation and Advanced Technology Research Program of Tianjin, China (Grant no. 12JCQNJC02100).

\section{References}

[1] P. S. Stephanou and V. G. Mavrantzas, "Quantitative predictions of the linear viscoelastic properties of entangled polyethylene and polybutadiene melts via modified versions of modern tube models on the basis of atomistic simulation data," Journal of Non-Newtonian Fluid Mechanics, vol. 200, pp. 111-130, 2013.

[2] I. M. Balashova, R. G. a Buduen, and R. P. Danner, "Solubility of organic solvents in 1, 4-cis-polybutadiene," Fluid Phase Equilibria, vol. 334, pp. 10-14, 2012.

[3] V. K. Srivastava, M. Maiti, and R. V. Jasra, "Synthesis and utilization of alternative chain transfer agent in cobalt catalyzed 1,3-butadiene polymerization reaction to produce cispolybutadiene rubber," European Polymer Journal, vol. 47, no. 12, pp. 2342-2350, 2011.

[4] M. M. Afiq and A. R. Azura, "Effect of sago starch loadings on soil decomposition of natural rubber latex (NRL) composite films mechanical properties," International Biodeterioration \& Biodegradation, vol. 85, pp. 139-149, 2013.

[5] S. S. Sarkawi, W. K. Dierkes, and J. W. M. Noordermeer, "The influence of non-rubber constituents on performance of 
silica reinforced natural rubber compounds," European Polymer Journal, vol. 49, no. 10, pp. 3199-3209, 2013.

[6] T. S. Anirudhan, P. L. Divya, and J. Nima, "Silylated montmorillonite based molecularly imprinted polymer for the selective binding and controlled release of thiamine hydrochloride," Reactive and Functional Polymers, vol. 73, no. 8, pp. 1144-1155, 2013.

[7] K. Fukushima, M. Wu, S. Bocchini, A. Rasyida, and M. Yang, "PBAT based nanocomposites for medical and industrial applications," Materials Science and Engineering C, vol. 32, no. 6, pp. 1331-1351, 2012.

[8] A. C. Lopes, J. C. C. Ferreira, C. M. Costa, and S. LancerosMéndez, "Crystallization kinetics of montmorillonite/ poly(vinylidene fluoride) composites and its correlation with the crystalline polymer phase formation," Thermochimica Acta, vol. 574, pp. 19-25, 2013.

[9] M. El Achaby, H. Ennajih, F. Z. Arrakhiz et al., "Modification of montmorillonite by novel geminal benzimidazolium surfactant and its use for the preparation of polymer organoclay nanocomposites," Composites Part B, vol. 51, pp. 310-317, 2013.

[10] C. Oliveira and J. Rubio, "Kaolin aerated flocs formation assisted by polymer-coated microbubbles," International Journal of Mineral Processing, vol. 106-109, pp. 31-36, 2012.

[11] C. Z. Hu, G. X. Chen, H. J. Liu, H. Zhao, and J. H. Qu, "Characterization of flocs generated by preformed and in situ formed $\mathrm{Al}_{13}$ polymer," Chemical Engineering Journal, vol. 197, pp. 10-15, 2012.

[12] A. Middea, T. L. A. P. Fernandes, R. Neumann, O. F. M. Gomes, and L. S. Spinelli, "Evaluation of Fe(III) adsorption onto palygorskite surfaces," Applied Surface Science, vol. 282, pp. 253-258, 2013.

[13] W. C. Yan, P. Yuan, M. Chen, L. J. Wang, and D. Liu, "Infrared spectroscopic evidence of a direct addition reaction between palygorskite and pyromellitic dianhydride," Applied Surface Science, vol. 265, pp. 585-590, 2013.

[14] X. Z. Li, X. W. Lu, Y. Q. Meng, C. Yao, and Z. G. Chen, "Facile synthesis and catalytic oxidation property of palygorskite/mesocrystalline $\mathrm{Ce}_{1-x} \mathrm{Mn}_{x} \mathrm{O}_{2}$ nanocomposites," Journal of Alloys and Compounds, vol. 562, pp. 56-63, 2013.

[15] W. C. Yan, D. Liu, D. Y. Tan, P. Yuan, and M. Chen, "FTIR spectroscopy study of the structure changes of palygorskite under heating," Spectrochimica Acta Part A, vol. 97, pp. 10521057, 2012.

[16] H. B. Liu, T. H. Chen, D. Y. Chang et al., "Effect of rehydration on structure and surface properties of thermally treated palygorskite," Journal of Colloid and Interface Science, vol. 393, pp. 87-91, 2013.

[17] Q. Q. Xie, T. H. Chen, H. Zhou et al., "Mechanism of palygorskite formation in the red clay formation on the Chinese loess plateau, northwest China," Geoderma, vol. 192, pp. 39-49, 2013.

[18] S. X. Zuo, C. Yao, W. J. Liu et al., "Preparation of ureido-palygorskite and its effect on the properties of urea-formaldehyde resin," Applied Clay Science, vol. 80-81, pp. 133-139, 2013.

[19] Y. Shen and A. C. Lua, "Structural and transport properties of BTDA-TDI/MDI co-polyimide (P84)-silica nanocomposite membranes for gas separation," Chemical Engineering Journal, vol. 188, pp. 199-209, 2012.

[20] L. J. Zeng, R. Wang, L. H. Zhu, and J. D. Zhang, "Graphene and CdS nanocomposite: a facile interface for construction of DNA-based electrochemical biosensor and its application to the determination of phenformin," Colloids and Surfaces B, vol. 110, pp. 8-14, 2013.

[21] J. K. G. Bunk, A. Drechsler, S. Rauch, P. Uhlmann, M. Stamm, and R. Rennekamp, "The distribution of hydrophobized inorganic nanoparticles in thermoresponsive polymer nanocomposite films investigated by scanning probe and electron microscopy," European Polymer Journal, vol. 49, no. 8, pp. 19942004, 2013

[22] D. B. Mahadik, A. V. Rao, A. P. Rao, P. B. Wagh, S. V. Ingale, and S. C. Gupta, "Effect of concentration of trimethylchlorosilane (TMCS) and hexamethyldisilazane (HMDZ) silylating agents on surface free energy of silica aerogels," Journal of Colloid and Interface Science, vol. 356, no. 1, pp. 298-302, 2011.

[23] X. Dong, Q. Y. Zong, and J. X. He, "Anisotropic surface properties and wettability of disperse dye single crystal," Dyes and Pigments, vol. 96, no. 3, pp. 636-641, 2013.

[24] N. Eshtiaghi and K. P. Hapgood, "A quantitative framework for the formation of liquid marbles and hollow granules from hydrophobic powders," Powder Technology, vol. 223, pp. 65-76, 2012.

[25] D. J. Woo, B. Sneed, F. Peerally et al., "Synthesis of nanodiamond-reinforced aluminum metal composite powders and coatings using high-energy ball milling and cold spray," Carbon, vol. 63, pp. 404-415, 2013.

[26] S. M. Mirabedini and A. Kiamanesh, "The effect of micro and nano-sized particles on mechanical and adhesion properties of a clear polyester powder coating," Progress in Organic Coatings, vol. 76, no. 11, pp. 1625-1632, 2013.

[27] N. Wang, Q. Fang, J. Zhang, E. Chen, and X. Zhang, "Incorporation of nano-sized mesoporous MCM-41 material used as fillers in natural rubber composite," Materials Science and Engineering A, vol. 528, no. 9, pp. 3321-3325, 2011.

[28] S. Kango, S. Kalia, A. Celli, J. Njuguna, Y. Habibi, and R. Kumar, "Surface modification of inorganic nanoparticles for development of organic-inorganic nanocomposites-a review," Progress in Polymer Science, vol. 38, no. 8, pp. 1232-1261, 2013.

[29] S. M. Mirabedini and A. Kiamanesh, "The effect of micro and nano-sized particles on mechanical and adhesion properties of a clear polyester powder coating," Progress in Organic Coatings, vol. 76, no. 11, pp. 1625-1632, 2013.

[30] A. Sobolkina, V. Mechtcherine, V. Khavrus et al., "Dispersion of carbon nanotubes and its influence on the mechanical properties of the cement matrix," Cement and Concrete Composites, vol. 34, no. 10, pp. 1104-1113, 2012.

[31] L. C. Tang, Y. J. Wan, D. Yan et al., “The effect of graphene dispersion on the mechanical properties of graphene/epoxy composites," Carbon, vol. 60, pp. 16-27, 2013. 

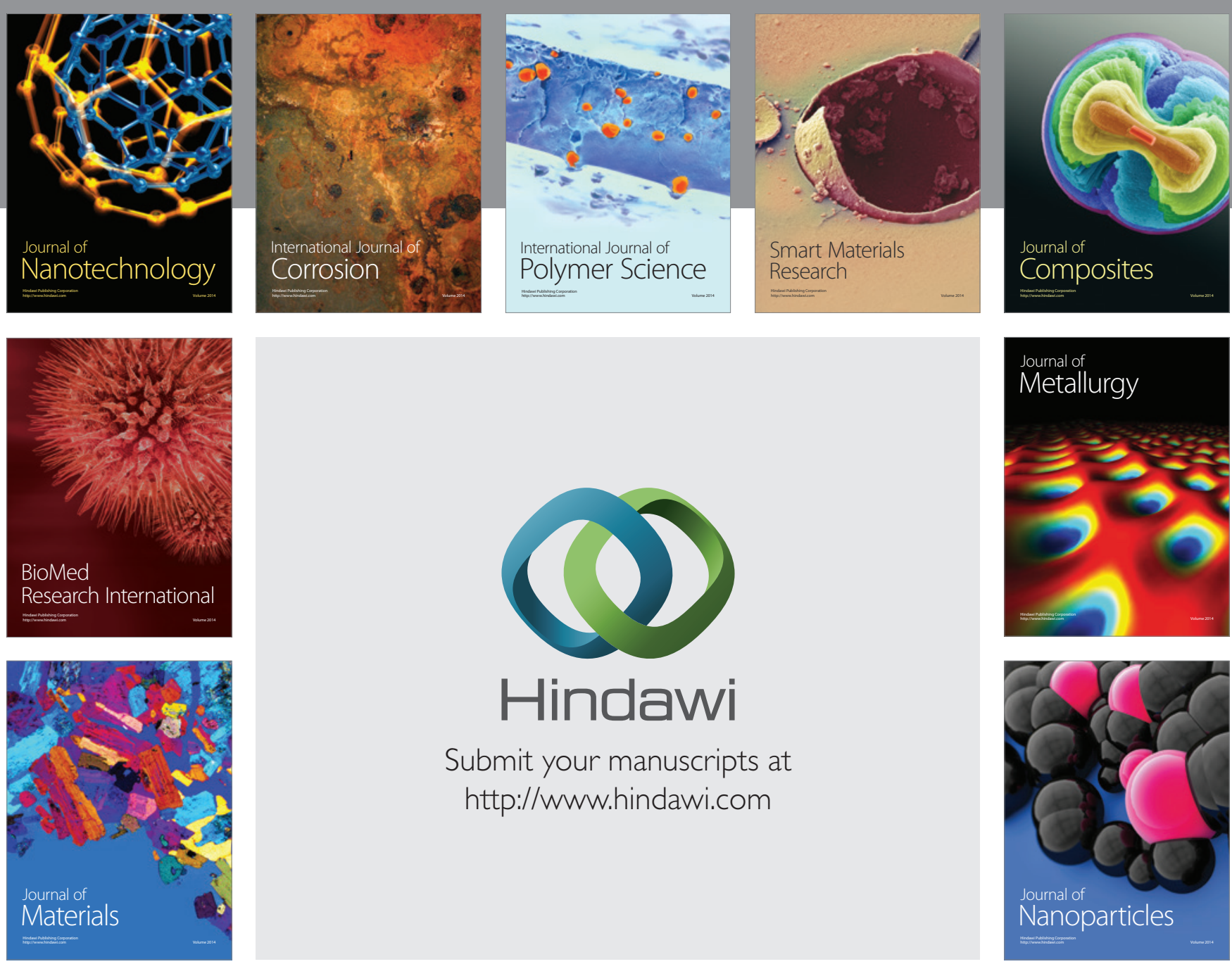

Submit your manuscripts at http://www.hindawi.com
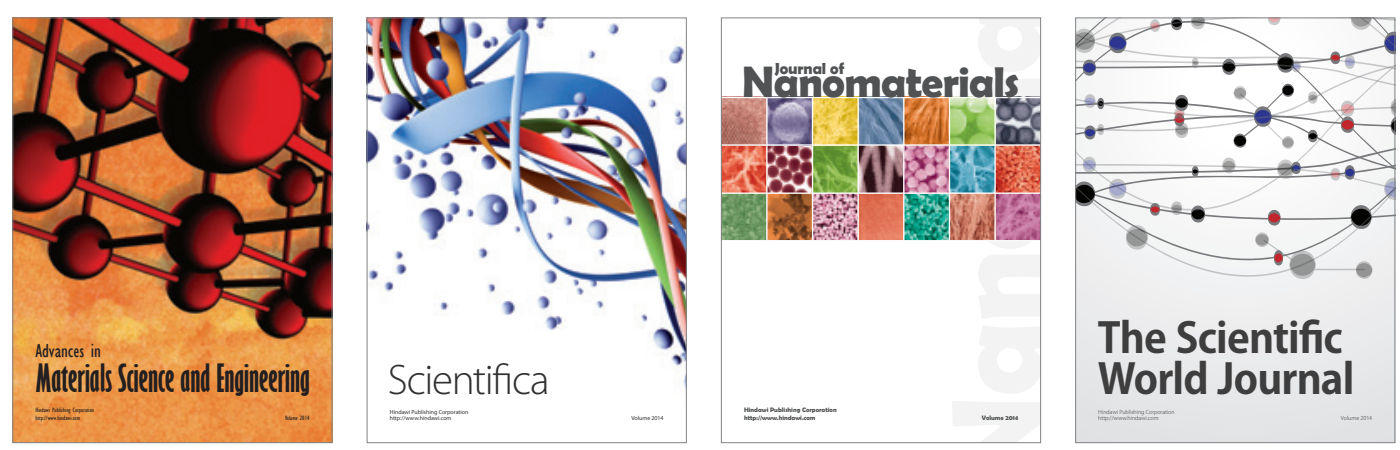

\section{The Scientific World Journal}
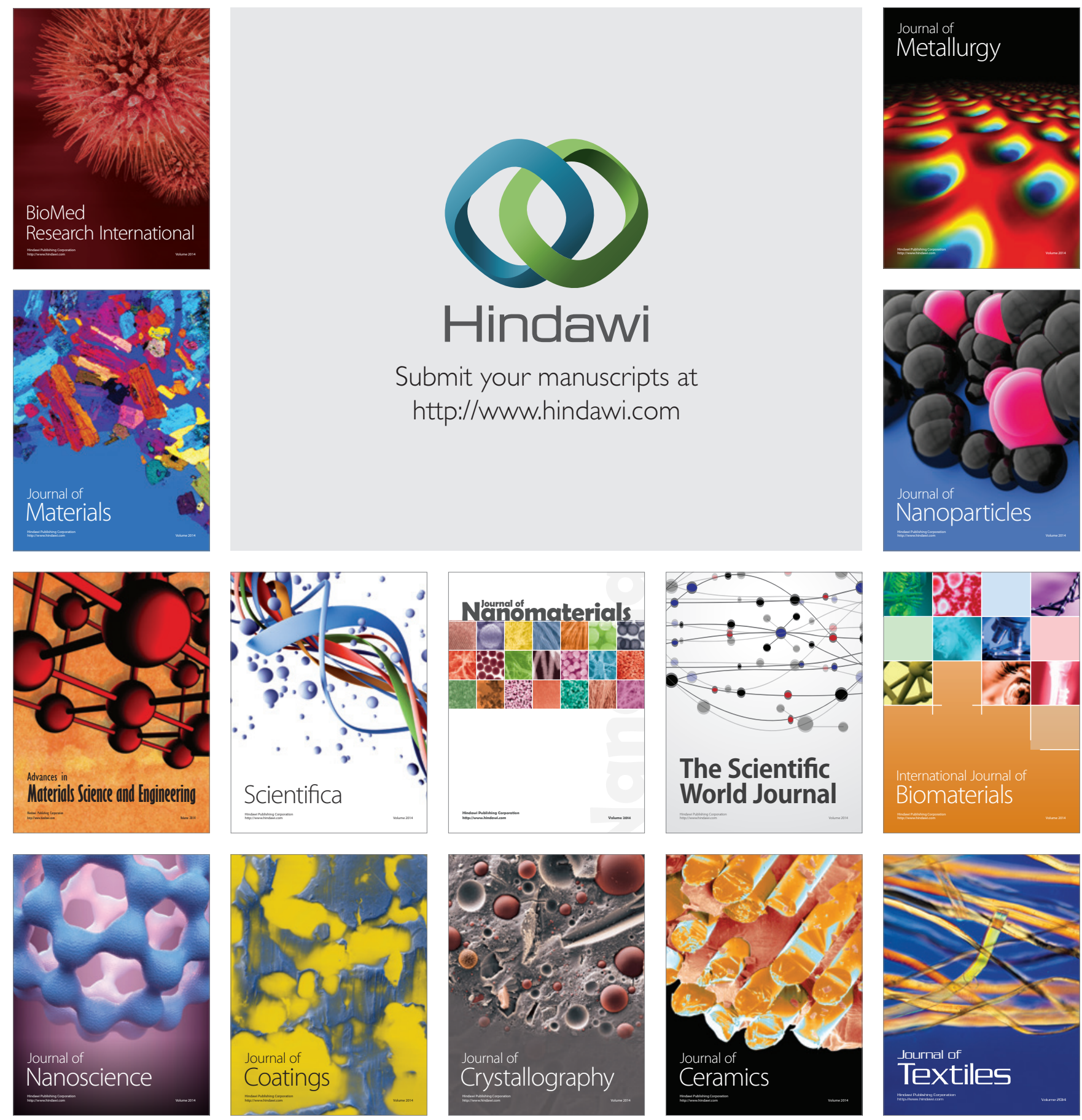